\title{
Long-range exchange bias through a metal spacer
}

\author{
Yuan-Jen Lee ${ }^{\mathrm{a}, *}$, Ching-Ray Chang ${ }^{\mathrm{a}}$, Tzay-Ming Hong ${ }^{\mathrm{b}}$, \\ C.H. Ho ${ }^{\mathrm{a}}$, Minn-Tsong Lin $^{\mathrm{a}}$ \\ a Department of Physics, National Taiwan University, 1. Section 4 Roosevelt Road, Taipei Taiwan \\ ${ }^{\mathrm{b}}$ Department of Physics, National Tsing Hua University, Hsinchu, Taiwan
}

\begin{abstract}
We have theoretically analyzed the long-range exchange bias between a ferromagnet and an antiferromagnet separated by a nonmagnetic metal spacer. The Fermi-Dirac distribution was included in the Ruderman-KittelKasuya-Yosida interaction to study the thermal effects of the conduction electrons, and thus to study the temperature effects and thickness dependence in the trilayer structure. The experimentally observed oscillatory exchange bias through the metal spacer is in good agreement with the calculated results. (C) 2002 Elsevier Science B.V. All rights reserved.
\end{abstract}

Keywords: Exchange bias; Exchange coupling, - RKKY; Dipolar interactions

The hysteresis loop displacement was first discovered in 1956 by Meiklejohn and Bean [1,2], when they were studying fine particles of cobalt (ferromagnet) with the cobaltous oxide (antiferromagnet) shell. This displacement was known as the exchange bias $\left(H_{\mathrm{E}}\right)$, and the origin of the $H_{\mathrm{E}}$ is a consequence of the interaction between a ferromagnetic (FM) and antiferromagnetic (AFM) layer at interface. On the other hand, the longrange magnetic coupling between two FM layers across a nonmagnetic conduction metal (M) has attracted a lot of attention in the past decade [3-7]. The interlayer exchange coupling between two FM layers behaves oscillatory with the thickness of the metal spacer, and this oscillatory behavior has been explained successfully based on the Ruderman-Kittel-Kasuya-Yosida (RKKY) model [6,7]. The FM/M/AFM trilayer system was first studied by Gökemeijer et al. in 1997 [8]; in their results, the $H_{\mathrm{E}}$ can be measured, too. But the interlayer exchange coupling decays exponentially with increasing thickness of the metal spacer, instead of an oscillatory coupling as in the FM/M/FM trilayer system. Recently, several experiments showed that the oscillation of the long-range $H_{\mathrm{E}}$ was observed [9-11], e.g., in $\mathrm{NiFe} / \mathrm{Cu} /$

\footnotetext{
*Corresponding author. Fax: + 886-2-3366-5167.

E-mail address: crchang9@phys.ntu.edu.tw (Y.-J. Lee).
}

$\mathrm{NiO}$ structures. Fig. 1 shows the $H_{\mathrm{E}}$ in $\mathrm{NiFe}(100 \AA) / \mathrm{Cu} /$ $\mathrm{NiO}(250 \AA)$ as a function of $\mathrm{Cu}$ thickness at different measured temperatures. One finds that the long-range $H_{\mathrm{E}}$ oscillates with $\mathrm{Cu}$ thickness, and the interlayer exchange coupling strongly depends on the temperature [11].

Here, we theoretically analyze this oscillation of the $H_{\mathrm{E}}$ in $\mathrm{NiFe}(100 \AA) / \mathrm{Cu} / \mathrm{NiO}(250 \AA)$ in detail. The RKKY interaction and the dipolar interaction are used to calculate the temperature effects and thickness dependence of the $H_{\mathrm{E}}$ in this trilayer system. In our model the interfacial spin in the AFM layer is assumed to be uncompensated, therefore there is a net magnetic moment $\left(\vec{m}_{\mathrm{AF}}\right)$ in the AFM at the interfaces. For simplicity, we assumed that the FM anisotropy axis, AFM anisotropy axis, and the applied field are in the same direction, but the $\vec{m}_{\mathrm{AF}}$ and the cooling field are along the opposite direction. The energy per unit area in the $\mathrm{FM} / \mathrm{M} / \mathrm{AFM}$ system can then be written as (Fig. 2)

$$
\begin{aligned}
\varepsilon(t)= & K_{\mathrm{F}} t_{\mathrm{F}} \sin ^{2} \theta-H M_{\mathrm{F}} t_{\mathrm{F}} \cos \theta \\
& +\varepsilon_{\mathrm{dip}}(t)+\varepsilon_{\mathrm{RKKY}}(t),
\end{aligned}
$$

where $t$ is the thickness of metal, $K_{\mathrm{F}}$ is the anisotropy of the FM layer, $t_{\mathrm{F}}$ is the thickness of the FM layer, $H$ is the applied field, and $\theta$ is the angle between $\vec{M}_{\mathrm{F}}$ and the 


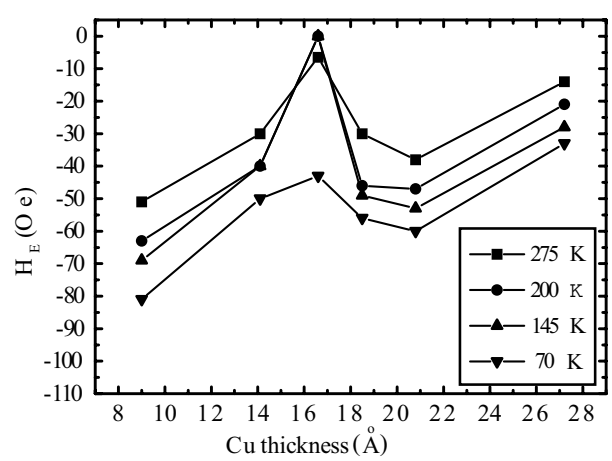

Fig. 1. The $H_{\mathrm{E}}$ values in $\mathrm{NiFe}(100 \AA) / \mathrm{Cu} / \mathrm{NiO}(250 \AA)$ as a function of $\mathrm{Cu}$ thickness at different measured temperature of $70,145,200$, and $275 \mathrm{~K}$.

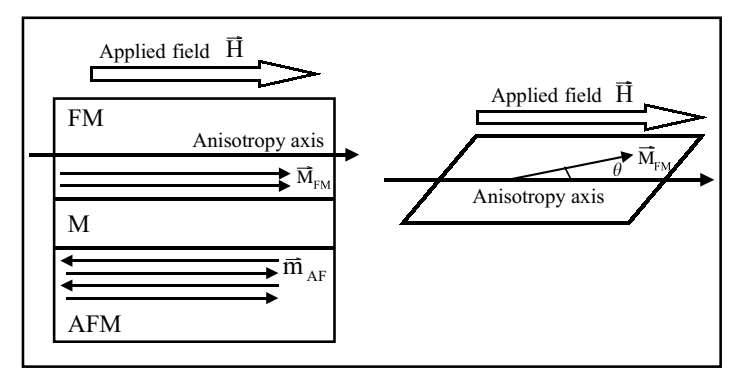

Fig. 2. Schematic diagram of the structure of $\mathrm{FM} / \mathrm{M} / \mathrm{AFM}$ trilayer system.

anisotropy axis. The first term in Eq. (1) accounts for the effect of the FM anisotropy, the second term is the effect of the applied field on the FM layer,

$$
\begin{aligned}
\varepsilon_{\mathrm{dip}}(t) & =\frac{\vec{M}_{\mathrm{F}} \cdot \vec{m}_{\mathrm{AF}}-3\left(\vec{M}_{\mathrm{F}} \cdot \hat{n}\right)\left(\vec{m}_{\mathrm{AF}} \cdot \hat{n}\right)}{t^{3}} t_{\mathrm{F}} \\
& \approx-\frac{1}{t^{3}} M_{\mathrm{F}} m_{\mathrm{AF}} t_{\mathrm{F}} \cos \theta
\end{aligned}
$$

is the dipolar interaction between $\vec{M}_{\mathrm{F}}$ and $\vec{m}_{\mathrm{AF}}$. The indirect exchange coupling (RKKY interaction) between $\vec{M}_{\mathrm{F}}$ and $\vec{m}_{\mathrm{AF}}$ at zero temperature [12] is

$$
\begin{aligned}
\varepsilon(t)= & \frac{4 J^{2} m^{*} k_{\mathrm{f}}^{4}}{(2 \pi)^{3}} \times \frac{2 k_{\mathrm{f}} t \cos 2 k_{\mathrm{f}} t-\sin 2 k_{\mathrm{f}} t}{\left(2 k_{\mathrm{f}} t\right)^{4}} \\
& \times\left(-M_{\mathrm{F}} m_{\mathrm{AF}} t_{\mathrm{F}} \cos \theta\right) .
\end{aligned}
$$

To obtain the exchange coupling between FM and AFM planes, we integrate over spins in the FM and AFM planes.

$$
\begin{aligned}
\varepsilon_{\mathrm{RKKY}}(t) \approx & \frac{J^{2} m k_{\mathrm{f}}^{2}}{4 \pi^{2} \hbar^{2}}\left[\frac{2 k_{\mathrm{f}} t \cos 2 k_{\mathrm{f}} t-\sin 2 k_{\mathrm{f}} t}{\left(2 k_{\mathrm{f}} t\right)^{2}}\right. \\
& \left.-\int_{2 k_{\mathrm{f}} t}^{\infty} \frac{\sin y}{y} \mathrm{~d} y\right] \times\left(-M_{\mathrm{F}} m_{\mathrm{AF}} t_{\mathrm{F}} \cos \theta\right),
\end{aligned}
$$

is the indirect exchange coupling (RKKY interaction) between FM and AFM planes at zero temperature [13].
It should be noted that the unit of $\varepsilon_{\text {dip }}$ and $\varepsilon_{\mathrm{RKKY}}$ is the energy per unit area. In order to study the influence of temperature dependence in the long-range $H_{\mathrm{E}}$, we include the Fermi-Dirac distribution in the RKKY interaction. For the low temperature limit,

$$
\begin{aligned}
\varepsilon_{\mathrm{RKKY}}(t, T) \approx & \varepsilon_{\mathrm{RKKY}}(t) \\
& +\frac{J^{2} m^{3} k_{\mathrm{B}}^{2}}{12 \hbar^{6} k_{\mathrm{f}}^{2}}\left(\int_{2 k_{\mathrm{f}} t}^{\infty} \frac{\sin x}{x} \mathrm{~d} x\right) \\
& \times\left(-M_{\mathrm{F}} m_{\mathrm{AF}} t_{\mathrm{F}} \cos \theta\right) T^{2},
\end{aligned}
$$

where $k_{\mathrm{B}}$ is Boltzmann's constant, and $k_{\mathrm{f}}$ is the Fermi wave vector. And we let

$m_{\mathrm{AF}}(T)=m_{0}\left(1-b T^{2}\right)$

to $m_{\mathrm{AF}}$ [14]. Therefore,

$$
\begin{aligned}
\varepsilon(t, T)= & K_{\mathrm{F}} t_{\mathrm{F}} \sin ^{2} \theta-H M_{\mathrm{F}} t_{\mathrm{F}} \cos \theta \\
& +\varepsilon_{\text {dip }}(t, T)+\varepsilon_{\mathrm{RKKY}}(t, T) .
\end{aligned}
$$

Minimizing the energy with respect to $\theta$, the displacement of hysteresis loop is found to be

$$
\begin{aligned}
H_{\mathrm{E}}= & \frac{\varepsilon_{\mathrm{dip}}(t, T)+\varepsilon_{\mathrm{RKKY}}(t, T)}{M_{\mathrm{F}} t_{\mathrm{F}} \cos \theta} \\
= & -\left\{\frac{1}{t^{3}}+\frac{J^{2} m k_{\mathrm{f}}^{2}}{4 \pi^{2} \hbar^{2}}\left[\frac{2 k_{\mathrm{f}} t \cos 2 k_{\mathrm{f}} t-\sin 2 k_{\mathrm{f}} t}{\left(2 k_{\mathrm{f}} t\right)^{2}}\right.\right. \\
& \left.\left.-\int_{2 k_{\mathrm{f}}}^{\infty} \frac{\sin y}{y} \mathrm{~d} y\right]+\frac{J^{2} m^{3} k_{\mathrm{B}}^{2}}{12 \hbar^{6} k_{\mathrm{f}}^{2}}\left(\int_{2 k_{\mathrm{f}}}^{\infty} \frac{\sin x}{x} \mathrm{~d} x\right) T^{2}\right\} \\
& \times m_{0}\left(1-b T^{2}\right),
\end{aligned}
$$

where $m_{0}$ and $b$ are our fitting parameter. Fig. 3 exhibits results for Eq. (8) at different temperature, where $m_{0}$ is $6 \times 10^{-19} \mathrm{emu}$, and $b$ is $1 \times 10^{-5} \mathrm{~K}^{-2}$. We have also analyzed experimental data with different thickness of $\mathrm{NiO}$, and it shows $m_{0}$ is $6 \times 10^{-20}$, and $b$ is $4 \times 10^{-5}$ [15]. It means that there is about 60 bohr magneton at interfaces in AFM layer at zero temperature, and as the temperature increases, the $\vec{m}_{\mathrm{AF}}$ rotates with $\mathrm{AFM}$ anisotropy. As the temperature increases the AFM layer

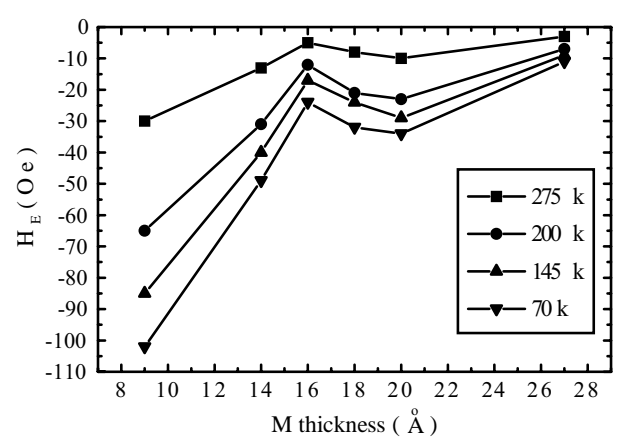

Fig. 3. Temperature dependence of the calculated $H_{\mathrm{E}}$ in FM/ $\mathrm{M} / \mathrm{AFM}$ for different thicknesses of the metal spacer. Here $k_{\mathrm{f}}$ is $\pi / 10 \AA^{-1}$. 
approaches the paramagnetic state, so the $\vec{m}_{\mathrm{AF}}$ couples weakly with the neighbor spin within the AFM layer. We find that the RKKY interaction competes with the dipolar interaction, and the oscillation only becomes significant as the RKKY interaction dominates. As temperature increases, the $H_{\mathrm{E}}$ becomes oscillatory with variation of the film thickness, for the dominant contribution from the RKKY interaction.

In summary, we have analyzed the long-range oscillation $H_{\mathrm{E}}$ in $\mathrm{FM} / \mathrm{M} / \mathrm{AFM}$ trilayer system. The results show the interlayer exchange coupling is strongly dependent on the temperature, and the $H_{\mathrm{E}}$ oscillates with the thickness of the metal as temperature increases. The experimentally observed oscillatory $H_{\mathrm{E}}$ through the metal spacer is in good agreement with the calculated results. It needs to be pointed out that the uncompensated moment $m_{\mathrm{AF}}$ increases with the increase of the $\mathrm{NiO}$ thickness.

\section{References}

[1] W.H. Meiklejohn, C.P. Bean, Phys. Rev. 102 (1956) 1413.

[2] W.H. Meiklejohn, C.P. Bean, Phys. Rev. 105 (1957) 904.
[3] S.S.P. Parkin, N. More, K.P. Roche, Phys. Rev. Lett. 64 (1990) 2304.

[4] Y. Wang, P.M. Levy, J.L. Fry, Phys. Rev. Lett. 65 (1990) 2732.

[5] S.S.P. Parkin, R. Bhadra, K.P. Roche, Phys. Rev. Lett. 66 (1991) 2152.

[6] W. Baltensperger, J.S. Helman, Appl. Phys. Lett. 57 (1990) 2954.

[7] P. Bruno, C. Chappert, Phys. Rev. Lett. 67 (1991) 1602.

[8] N.J. Gökemeijer, T. Ambrose, C.L. Chien, Phys. Rev. Lett. 79 (1997) 4270.

[9] L. Thomas, A.J. Kellock, S.S.P. Parkin, J. Appl. Phys. 87 (2000) 5061.

[10] T. Mewes, B.F.P. Roos, S.O. Demokritov, B. Hillebrands, J. Appl. Phys. 87 (2000) 5064.

[11] Minn-Tsong Lin, C.H. Ho, Ching-Ray Chang, Y.D. Yao, Phys. Rev. B 63 (2001) 100,404(R).

[12] C. Kittel, Quantum Theory Solids, Wiley, New York, 1963, p. 364 (Chapter 18).

[13] W. Baltensperger, J.S. Helman, Appl. Phys. Lett. 57 (1990) 2954.

[14] Z.Q. Qiu, J.E. Mattson, C.H. Sowers, U. Welp, S.D. Bader, H. Tang, J.C. Walker, Phys. Rev. B 45 (1992) 2252.

[15] Yuan-Jen Lee, Ching-Ray Chang, Tzay-Ming Hong, C.H. Ho, Minn-Tsong Lin, to be published. 